\title{
Mucocele de apêndice: relato de caso
}

\author{
Mucocele of appendix: case report \\ Apéndice Mucocele: reporte de caso \\ Laura dos Reis CHALUB ${ }^{1}$ \\ Amanda Oliva SPAZIANI ${ }^{1}$ \\ Raissa Silva FROTA ${ }^{2}$ \\ Stephanie Tiosso Fontes MONTEIRO \\ João Carlos Bizinotto Leal de LIMA ${ }^{1}$ \\ Trícia Aline Ribeiro Pattini de SOUZA ${ }^{4}$ \\ Gustavo Rivelli LAMBOGLIA ${ }^{4}$ \\ Rodrigo João Lopes TAVEIROS ${ }^{4}$ \\ Marcos Rogério MARQUES ${ }^{4}$ \\ Ana Carolina Borges GORGA \\ André Fraga RUEDA ${ }^{6}$ \\ Clerson Rodrigues MANAIA ${ }^{6}$ \\ Cleidjane Furtado REZENDE ${ }^{6}$ \\ Vladimir Tarcísio Delfino de OLIVEIRA ${ }^{6}$
}

${ }^{I}$ Curso de Medicina, Universidade Brasil, UB, 15600-000, Fernandópolis, São Paulo, Brasil

${ }^{2}$ Curso de Medicina, Universidade de Rio Verde, UniRV, 75901-970, Goianésia, Goiás, Brasil

${ }^{3}$ Curso de Medicina, Universidade Metropolitana de Santos, UNIMES, 11045-002, Santos, São Paulo, Brasil

${ }^{4}$ Residente de Cirurgia Geral, Hospital Ensino Santa Casa de Misericórdia, SCF, 15600-000, Fernandópolis, São Paulo, Brasil

${ }^{5}$ Médico Cirurgião Geral, Hospital Beneficência Portuguesa de Ribeirão Preto, 14010-090, Ribeirão Preto, São Paulo, Brasil

${ }^{6}$ Médico Cirurgião Geral, Hospital Ensino Santa Casa de Misericórdia, SCF, 15600-000, Fernandópolis, São Paulo, Brasil

\section{Resumo}

Introdução: A mucocele do apêndice é um termo utilizado para descrever uma dilatação deste órgão, devido ao acúmulo de secreção mucoide, tornando o apêndice dilatado, de paredes finas e com uma massa cística no seu interior. Na maioria dos casos a mucocele é resultante de neoplasia que obstrui a luz apendicular. Relato de caso: Sexo feminino, 55 anos, branca. Procurou atendimento médico devido à dor abdominal de moderada intensidade há cinco anos. Refere que os sintomas iniciaram com dor em fossa ilíaca direita e região lombar há cinco anos, quando começou a tratar lombalgia. Nesse ano, buscou atendimento graças a piora da dor. $\mathrm{O}$ abdome estava globoso, flácido, doloroso a palpação superficial e profunda em fossa ilíaca direita com ruídos hidroaéreos presentes. Devido a sintomatologia foram solicitados um ultrassom de abdome total que evidenciou imagem cística tubuliforme em região anexial direita, de aspecto simples, de contornos regulares e conteúdo anecoico, medindo $13,3 \mathrm{~cm}$ x 5,2 cm x 4,3 cm, sem septos, calcificações ou fluxo ao Doppler e ressonância magnética da pelve que demonstrou formação cística alongada em região anexial, mergulhante na região anexial, apêndice cecal não individualizado. Foi realizada uma cirurgia exploradora que resultou em apendicectomia e o produto enviado para análise histopatológica que resultou em mucocele de apêndice, sem critérios histológicos de malignidade. As mucoceles de apêndice foram classificadas em 3 categorias: hiperplasia mucosa sem atipias, mucocele simples; cistadenoma mucinoso, com algum grau de atipia e cistadenocarcinomamucinoso, com invasão estromal ou implantes peritoniais. Conclusão: A sintomatologia é a dor abdominal, de intensidade e duração variáveis, podendo também ser observados a presença de massa palpável em fossa ilíaca direita e emagrecimento. Embora cerca de $25 \%$ dos casos sejam assintomáticos, alguns estudos demonstram a correlação entre sintomatologia exuberante e displasia Aproximadamente $20 \%$ dos casos de mucocele de apêndice têm associação com o câncer colorretal, no entanto, outras neoplasias como os carcinomas de ovário, endométrio, mama, próstata, bexiga e rim também podem estar presentes.

Descritores: Mucocele; Apêndice; Dor Abdominal.

\section{Abstract}

Introduction: The mucocele of the appendix is a term used to describe a dilatation of this organ due to the accumulation of mucoid secretion, making the appendix dilated, thin-walled and with a cystic mass inside. In most cases, the mucocele is the result of neoplasia. that obstructs the appendicular light. Case Report: Female, 55 years old, white. She sought medical attention due to moderate abdominal pain five years ago. He states that the symptoms started with pain in the right iliac fossa and lower back five years ago, when he started treating low back pain. This year, sought care thanks to worsening pain. The abdomen was globose, flaccid, painful to superficial and deep palpation in the right iliac fossa with airborne noises present. Due to the symptoms, a total abdomen ultrasound was requested, which showed a tubuliform cystic image in the right adnexal region, with simple appearance, regular contours and anechoic content, measuring $13.3 \mathrm{~cm} \times 5.2 \mathrm{~cm}$ x $4.3 \mathrm{~cm}$, without septa, calcifications or Doppler flow and magnetic resonance imaging of the pelvis that demonstrated elongated cystic formation in the adnexal region, submersion in the adnexal region, non-individualized caecal appendix. An exploratory surgery was performed which resulted in appendectomy and the product sent for histopathological analysis which resulted in appendiceal mucocele without histological criteria of malignancy. Appendix mucoceles were classified into 3 categories: mucosal hyperplasia without atypias, simple mucocele; mucinous cystadenoma, with some degree of atypia, and mucinous cystadenocarcinoma, with stromal invasion or peritoneal implants. Conclusion: The symptoms are abdominal pain of varying intensity and duration, and the presence of a palpable mass in the right iliac fossa and weight loss can also be observed. Although about $25 \%$ of cases are asymptomatic, some studies show the correlation between exuberant symptomatology and dysplasia. Approximately $20 \%$ of cases of appendiceal mucocele are associated with colorectal cancer; however, other cancers such as ovarian, endometrium, breast, prostate, bladder and kidney carcinomas may also be present.

Descriptors: Mucocele; Appendix; Abdominal Pain.

\section{Resumen}

Introducción: El mucocele del apéndice es un término utilizado para describir un agrandamiento de este órgano debido a la acumulación de secreción mucoide lo que hace que el apéndice se dilate, tenga paredes delgadas y una masa quística en el interior. En la mayoría de los casos, el mucocele es el resultado de una neoplasia. que obstruye la luz apendicular. Informe de Caso: Mujer, 55 años, blanca. Ella buscó atención médica debido al dolor abdominal moderado hace cinco años. Afirma que los síntomas comenzaron con dolor en la fosa ilíaca derecha y la espalda baja hace cinco años, cuando comenzó a tratar el dolor lumbar. Este año, buscó atención gracias al empeoramiento del dolor. El abdomen era globoso, flácido, doloroso a palpación superficial y profunda en la fosa ilíaca derecha con ruidos presentes en el aire. Debido a los síntomas, se solicitó una ecografía total del abdomen, que mostró una imagen quística tubuliforme en la región anexial derecha, con apariencia simple, contornos regulares y contenido anecoico, que mide $13.3 \mathrm{~cm} \times 5.2 \mathrm{~cm}$ x $4.3 \mathrm{~cm}$, sin septos, calcificaciones o Imagen de flujo Doppler y resonancia magnética de la pelvis que demostró formación quística alargada en la región anexa, inmersión en la región anexa, apéndice cecal no individualizado. Se realizó una cirugía exploratoria que resultó en apendicectomía y el producto se envió para análisis histopatológico que resultó en mucocele apendicular sin criterios histológicos de malignidad. Los mucoceles del apéndice se clasificaron en 3 categorías: hiperplasia de la mucosa sin atipias, mucocele simple; cistadenoma mucinoso, con cierto grado de atipia, y cistadenocarcinoma mucinoso, con invasión estromal o implantes peritoneales. Conclusión: Los síntomas son dolor abdominal de intensidad y duración variables, y también se puede observar la presencia de una masa palpable en la fosa ilíaca derecha y la pérdida de peso. Aunque alrededor del $25 \%$ de los casos son asintomáticos, algunos estudios muestran la correlación entre la sintomatología exuberante y la displasia. Aproximadamente el $20 \%$ de los casos de mucocele apendicular están asociados con el cáncer colorrectal; sin embargo, también pueden estar presentes otros cánceres como los carcinomas de ovario, endometrio, mama, próstata, vejiga y riñón.

Descriptores: Mucocele; Apéndice; Dolor Abdominal. 


\section{INTRODUÇÃO}

A mucocele de apêndice é uma rara patologia, com incidência de $0,2 \%$ a $0,4 \%$ de todas as apendicectomias, descrita inicialmente pelo austríaco Carl Freiherr von Rokitansky (1804-1878), em Handbook of Pathologic Anatomy (1842, vol. 2). R. Woodruff e J. R. McDonald, em Benign and Malignant Cystic Tumors of the Appendix (1940), classificaram a mucocele em dois tipos: o benigno e o maligno ${ }^{1}$.

O termo mucocele foi utilizado, por Feren, pela primeira vez em 1876, para descrever um apêndice alargado, com conteúdo gelatinoso; e está associado a três fatores: obstrução da luz apendicular, dilatação do apêndice devido ao acúmulo de secreção mucinosa e característica estéril da secreção ${ }^{1,2}$.

Inclui quatro entidades bem descritas: o tipo I é caracterizada por cistos de retenção, correspondente a obstrução simples do lúmen apendicular, geralmente por um fecalito e tem $20 \%$ de incidência de todas as mucoceles. No tipo II observa-se obstrução por hiperplasia da mucosa, com incidência de $5 \%$ a $25 \%$ das mucoceles, em quanto o tipo III corresponde à presença de neoplasia benigna ou cistoadenoma mucinoso, caracterizada por hiperplasia focal ou difusa da mucosa, preenchidas por material mucinoso. Histopatologicamente o tipo III mostra mucosa adenomatosa com epitélio mucinoso com núcleo basal e alterações displásicas mínimas. É a forma mais comum, ocorre em cerca de $60 \%$ dos casos de mucocele e em $0,07 \%$ a $0,3 \%$ das apendicectomias. Por fim, o tipo IV apresenta neoplasia maligna ou cistoadenocarcinoma mucinoso, representando de $11 \%$ a $20 \%$ dos casos de mucocele ${ }^{1-3}$.

Possui uma incidência maior no sexo feminino (4:1) e entre a quinta e sexta décadas de vida, porém casos incidentes na terceira década de vida também são descritos. Os achados clínicos e apresentação são inespecíficos ou assintomáticos e, em cerca de $50 \%$ dos casos, o diagnóstico préoperatório é difícil devido à falta de especificidade dos sintomas, por isso, acaba por ser um achado cirúrgico ou de exames de imagem solicitados por outras causas ${ }^{2,3}$.

Quando sintomático, o paciente pode queixar-se de dor em quadrante inferior direito, distensão abdominal e massa palpável ao exame físico ou autoexame, sendo a dor abdominal o sintoma mais comum. Febre, anorexia, náuseas, vômitos e perda ponderal podem estar associados, assim como sintomas urinários, de apendicite aguda e quadros ginecológicos. Além disso, a sintomatologia está mais associada a patologias malignas ${ }^{2-4}$.

Desde que as margens de ressecção sejam claras a apendicectomia é curativa para mucoceles apendiculares não rotas de origem benigna. Por outro lado, a cecectomia parcial pode ser indicada para uma mucocele benigna de base ampla que se projeta para a luz cecal. Se a parede cecal ou o íleo forem invadidos por tumor ou as margens cirúrgicas adequadas não puderem ser seguras, a ressecção ileocecal ou a hemicolectomia direita podem ser necessárias. No entanto, por causa da raridade, existe falta de diretrizes baseadas em evidências para o manejo dessas lesões ${ }^{4,5}$.

Em uma tomografia computadorizada préoperatória, a calcificação da parede da mucocele não é indicativa de malignidade, ao contrário do realce nodular da parede da mucocele. Além disso, estudos sugerem que a magnitude da mucocele pode refetir a malignidade. Mucoceles de origem benigna são geralmente menores que $2 \mathrm{~cm}$ de tamanho, enquanto as mucoceles devido a cistoadenoma ou cistoadenocarcinomas tendem a ser maiores que 2 $\mathrm{cm}$. No entanto, os exames histopatológicos são indispensáveis para determinar benignidade ou malignidade ${ }^{5}$.

\section{CASO CLÍNICO}

Paciente do sexo feminino, 55 anos de idade e cor branca, procurou atendimento médico devido à dor abdominal de moderada intensidade há cinco anos. Refere que os sintomas iniciaram com dor em fossa ilíaca direita e região lombar há cinco anos, de forma localizada, sem irradiação, sem fatores de melhora ou piora, quando começou a tratar a lombalgia. Nesse ano, buscou atendimento graças a piora da dor. Ao exame físico geral, estava em bom estado geral, corada, hidratada, acianótica, anictérica, eutrófica e nutrida, negando emagrecimento significativo em pouco espaço de tempo. Os exames cardiovascular e pulmonar apresentaram-se sem alterações.

O abdome estava globoso, flácido, doloroso a palpação superficial e profunda em fossa ilíaca direita com ruídos hidroaéreos presentes. Devido a sintomatologia foram solicitados um ultrassom de abdome total que evidenciou imagem cística tubuliforme em região anexial direita, de aspecto simples, de contornos regulares e conteúdo anecoico, medindo $13,3 \mathrm{~cm} \times 5,2 \mathrm{~cm} \times 4,3 \mathrm{~cm}$, sem septos, calcificações ou fluxo ao Doppler e ressonância magnética da pelve que demonstrou formação cística alongada em região anexial, mergulhante na região anexial, apêndice cecal não individualizado.

Foi realizada cirurgia exploradora que resultou em apendicectomia e o produto enviado para análise histopatológica resultou em mucocele de apêndice, sem critérios histológicos de malignidade (Figuras 1 a 5). A paciente obteve alta após o procedimento cirúrgico e retornou as atividades habituais em 45 dias, sem mais episódios álgicos. 


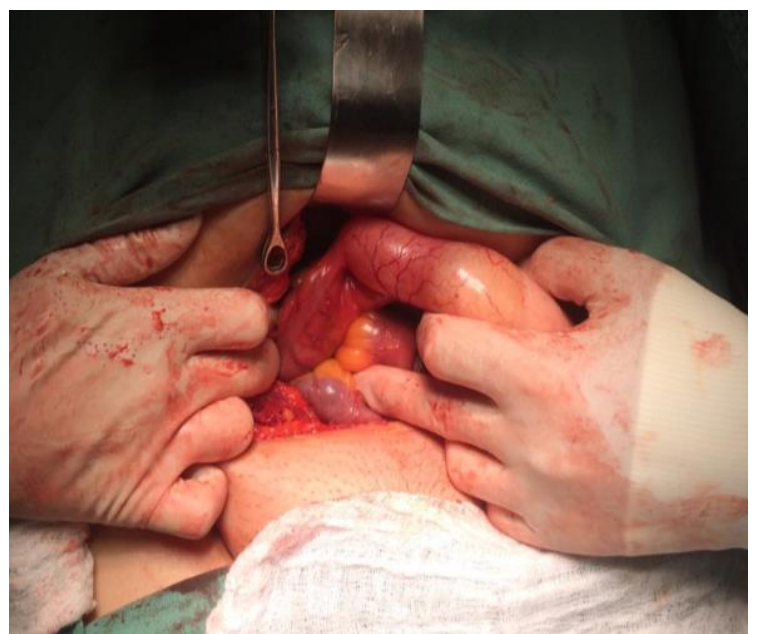

Figura 1: Campo visual da cavidade abdominal na cirurgia exploradora.

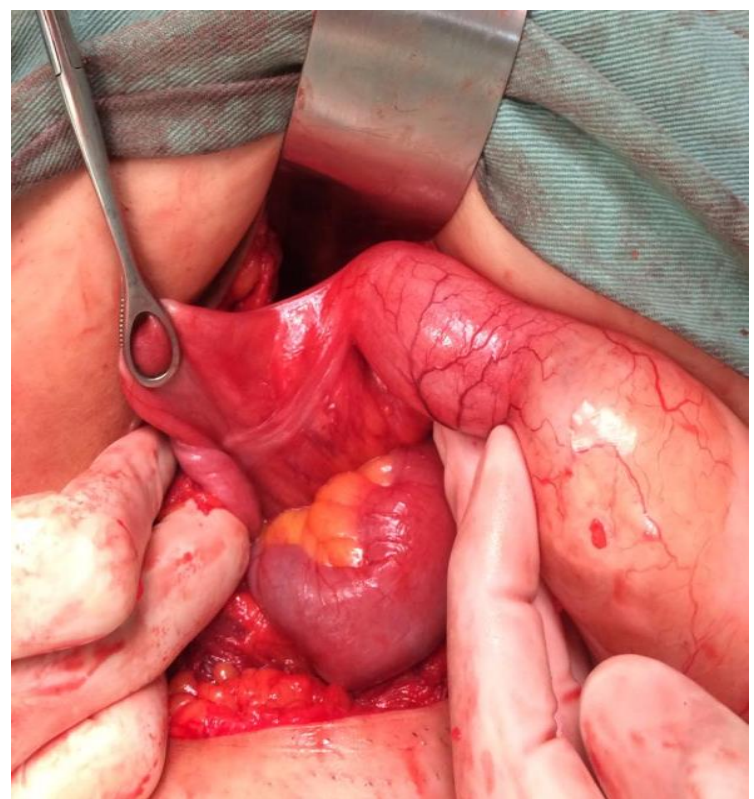

Figura 2: Exteriorização do apêndice cecal evidenciando a mucocele de apêndice.

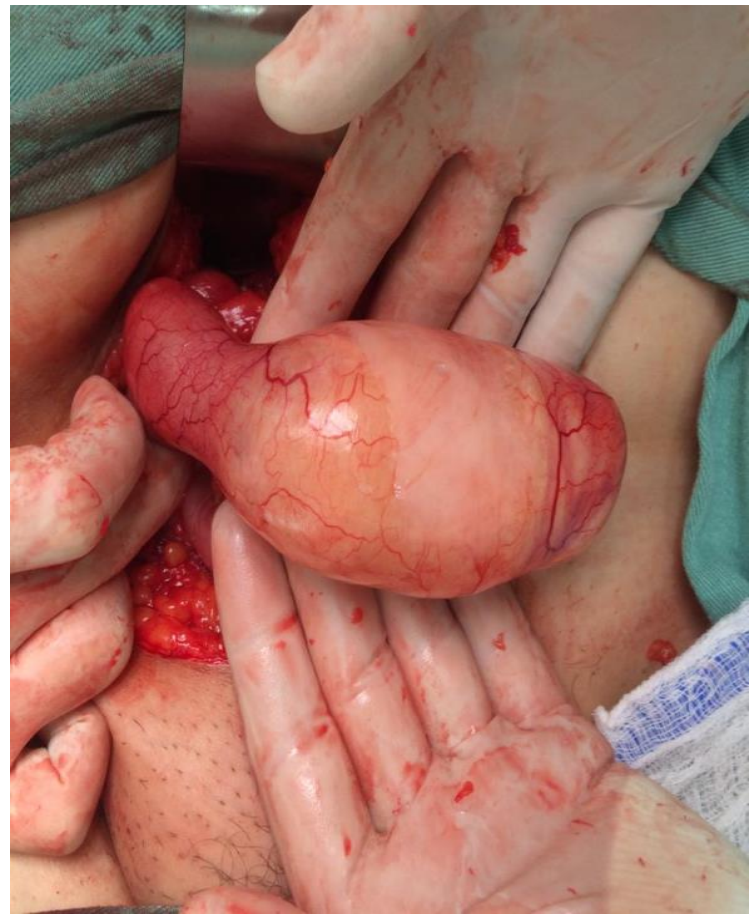

Figura 3: Mucocele de apêndice.

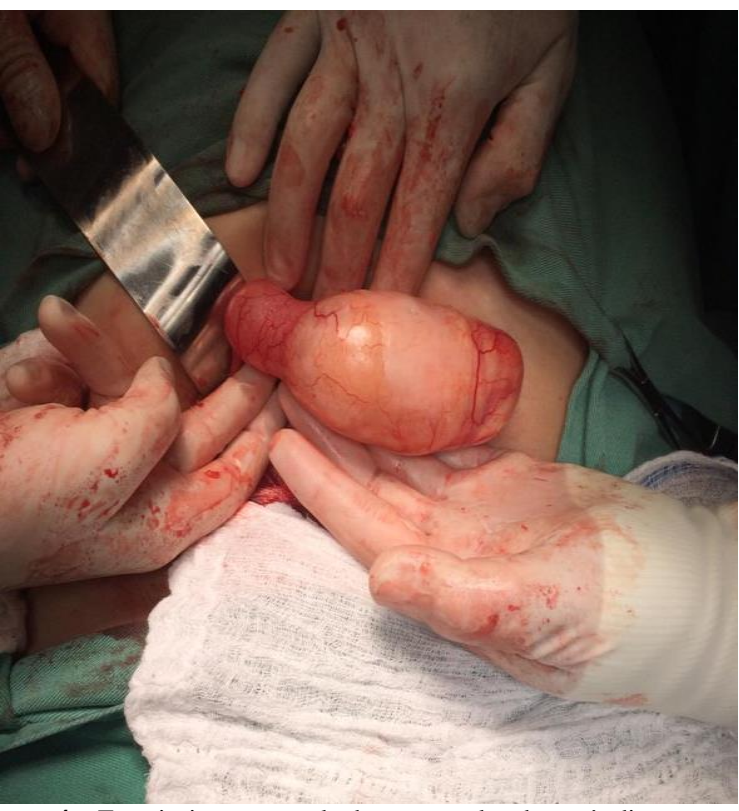

Figura 4: Exteriorização total da mucocele de apêndice antes da realização da apendicectomia.

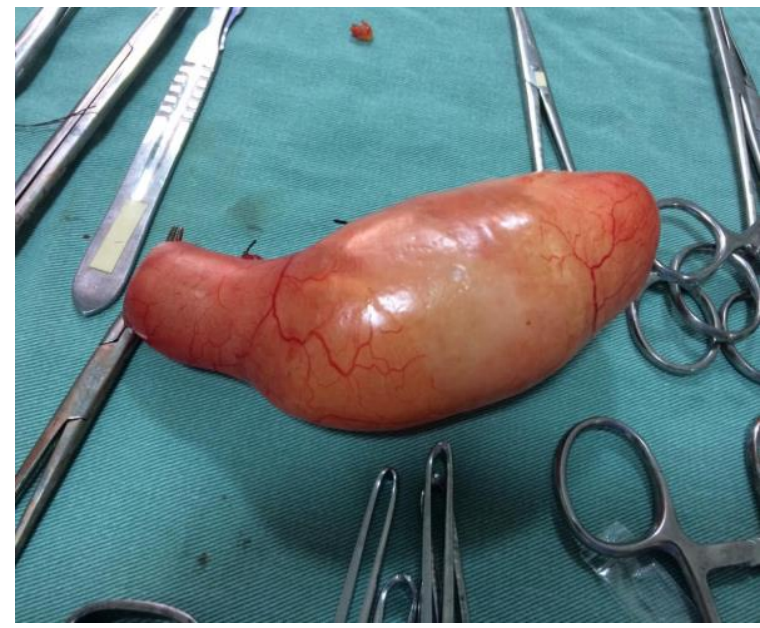

Figura 5: Mucocele de apêndice após apendicectomia

\section{DISCUSSÃO}

Devido a sua baixa incidência na população, pensa-se geralmente em outros diagnósticos diferenciais como: obstrução secundária por fecalito ocasionando mudanças epiteliais degenerativas, apendicite aguda, outras neoplasias benignas e malignas do apêndice, endometriose, cistos de ovário, abscesso, intussuscepção, pólipos e inversão de coto ${ }^{6}$. A sintomatologia não coopera para o diagnóstico précirúrgico e na maioria, os exames laboratoriais são bastante inespecíficos. Um estudo mostrou, em uma série de onze casos, leucocitose em sete pacientes, anemia em quatro pacientes (os quais necessitaram de transfusões sanguíneas) e alguns marcadores tumorais como o antígeno carcinoembrionário e o antígeno de câncer 19-9 presentes e normais em três pacientes. O que evidencia o valor genérico de testes laboratoriais, os quais não auxiliam diretamente a fechar um diagnóstico ${ }^{7}$.

Os exames de imagem como a tomografia computadorizada, ultrassonografia, clister opaco e colonoscopia, podem sugerir a presença de mucocele 
do apêndice, podendo auxiliar no diagnóstico préoperatório, proporcionam uma melhor qualidade no que diz respeito ao estudo anatômico da tumoração, mas ainda assim não correspondem a um diagnóstico fechado ${ }^{8}$.

Tendo em vista a dificuldade do diagnóstico pré-operatório, a ressecção cirúrgica é o tratamento de base. A apendicectomia aberta ou laparoscópica pode ser utilizada, principalmente em casos que evoluem com complicações. No entanto ainda não há consenso na utilização da cirurgia laparoscópica, pois algumas escolas defendem que a manipulação das pinças durante $\mathrm{o}$ procedimento $\mathrm{e}$ a maior probabilidade de ruptura do apêndice devido à técnica podem contribuir para a disseminação de células tumorais 9 .

Por ser uma patologia rara, com sintomatologia inespecífica e bastante variável, seu diagnóstico só é feito no intra-operatório e confirmado no pós-operatório. Por tal motivo a maioria das técnicas de ressecção utilizadas não é a adequada e deve-se ter bastante cuidado para não realizar uma apendicectomia isolada e permitir que células neoplásicas se disseminem piorando a condição de base do paciente ${ }^{10}$.

O prognóstico depende muito do subtipo histológico, mas em suma a sobrevida atinge a excelência, dentro da faixa de 91 a 100\% após a realização de apendicectomia para a retenção de cistos, hiperplasia mucoide ou cistoadenoma ${ }^{7}$.

\section{CONCLUSÃO}

A mucocele de apêndice é uma condição muito rara. Sua apresentação não segue apresentações sintomatológicas clássicas ou específicas, o que além de dificultar o diagnóstico pode levar a uma cirurgia exploradora sem sucesso, com disseminação de células neoplásicas e piora do estado basal do paciente. O diagnóstico além de difícil pode ser realizado acidentalmente durante o procedimento cirúrgico, o que aumenta a mortalidade, já que muitas vezes o cirurgião pode não estar usando a técnica adequada. Os exames de imagem podem proporcionar uma análise anatômica com maior acurácia, distinguindo os melhores procedimentos e técnicas a serem utilizadas para abordagem tumoral, evitando a ruptura do tecido friável e ressecção sem margens de segurança. A ressecção cirúrgica possui um alto potencial de cura, assim como também pode provocar complicações como o pseudomixoma peritonei, uma condição com alta morbimortalidade. Um adequado e precoce manejo clínico e cirúrgico é essencial para o bom desfecho do quadro.

\section{REFERÊNCIAS}

1. Ensuncho C, Osorio C, Marrugo Á, Herrera F. Obstrucción intestinal parcial producida por mucocele apendicular con fístula a íleon proximal. rev colomb cir. 2016;31(1):61-4.

2. Bichara DSJ, Luz Neto VC, Matuck MJ, Bichara DG. Pseudomixoma retroperitoneal (mucocele apendicular). Relatos Casos Cir. 2015;(1):1-3

3. Moré Cabrera JA, León Aulla SP, Pérez Zavala GA. Presentación inusual de mucocele apendicular. Acta Médica Centro. 2015;9(4):62-7.

4. Morano WF, Gleeson EM, Sullivan SH, Padmanaban V, Mapow BL, Shewokis PA et al. Clinicopathological features and management of appendiceal mucoceles: A systematic review. Am Surg. 2018;84(2):273-81.

5. Park KJ, Choi HJ, Kim SH. Laparoscopic approach to mucocele of appendiceal mucinous cystadenoma: Feasibility and short-term outcomes in 24 consecutive cases. Surg Endosc. 2015;29(11):3179-83.

6. Demetrashvili Z, Chkhaidze M, Khutsishvili K, Topchishvili G, Javakhishvili T, Pipia I et al. Mucocele of the appendix: case report and review of literature. Int Surg. 2012;97(3):266-69.

7. Basak F, Hasbahceci M, Yucel M, Sisik A, Acar A, Kilic A, Su Dur MS. Does it matter if it is appendix mucocele instead of appendicitis? Case series and brief review of literature. J Cancer Res Ther. 2018;14(6):1355-60.

8. Rabie ME, Al Shraim M, Al Skaini MS, Alqahtani S, El Hakeem I, Al Qahtani AS et al. Mucus containing cystic lesions "mucocele" of the appendix: the unresolved issues. Int J Surg Oncol. 2015;2015:139461.

9. Hao S, Lilly R, Bonatti HJR. Laparoscopic resection of an appendix mucocele in a breast cancer patient. Case Rep Surg. 2018; 2018:1780342.

10. Dixit A, Robertson JH, Mudan SS, Akle C. Appendiceal mucocoeles and pseudomyxoma peritonei. World J Gastroenterol. 2007; 13(16):2381-84.

\section{CONFLITO DE INTERESSES}

Os autores declaram não haver conflitos de interesse.

\section{AUTOR PARA CORRESPONDENCIA}

\section{Amanda Oliva Spaziani}

spazianimedicina@gmail.com
Submetido em 07/10/2019 Aceito em 07/11/2019 